\title{
PADRÃO DE CONFORMIDADE PARA UNIDADES DE INFORMAÇÃO: APRESENTAÇÃO DO MÓDELO INGLÊS
}

\section{Célia Regina Simonetti Barbalho}

\begin{abstract}
Resumo
Apresenta os parâmetros para a certificação de conformidade para Unidades de Informação, especificamente o da National Health Service da GrãBretanha.
\end{abstract}

Palavras-Chave

Qualidade; Certificação

\section{INTRODUÇÃO}

Os anos 90 têm se caracterizado pela globalização de economia e pelo surgimento de novos paradigmas gerenciais nas organizações. Estas mudanças são observadas através da maior participação social, da maior importância dada aos valores humanos, da busca pela satisfação das expectativas do clientes, da descentralização dos processos etc.

Em busca de novas tecnologias, novos mercados e novos métodos de gerenciamento ou redesenhamento dos processos surgiram novos modelos gerenciais oriundos de uma nova ordem econômica mundial chamada de "era da informação', onde as fontes de desenvolvimento são o conhecimento e o poder de comunicação.

Estes novos modelos de gerenciamento são, entre outros a Reengenharia, o Benchmarking, o TQM - Total Quality Manegement, o ABC - Custeio Baseado em Atividades, a Abordagem Estratégica, a Terceirização, a Quarteirização etc.

Dos modelos acima apresentados, o que tem causado maior impacto no cenário brasileiro é, sem dúvida alguma o da qualidade que pode ser entendida como um conjunto de procedimentos que iniciam com o conhecimento das necessidades e expectativas do cliente, influenciando na confecção original (projeto) de um produto ou serviço, bem como na sua confecção final, com o objetivo de cativar, manter e satisfazer o consumidor.

A discussão em torno dos aspectos inerentes aos processos de certificação de conformidade, tão próprio da gestão pela qualidade, bem como suas implicações para as Unidades de Informação é o objetivo desse trabalho que abordará o modelo de certificação inglês como parâmetro para adaptação às realidades nacionais.

\section{GESTÃO PELA QUALIDADE}

A gestão pela qualidade1 está diretamente relacionada com todos os outros modelos de gerenciamento na busca de soluções coerentes com os macroobjetivos das organizações, abrangendo todos os níveis hierárquicos, incluindo-se neste cenário as Unidades de Informação.

Tendo como um de seus princípios básicos a focalização nas necessidades do cliente, a gestão pela qualidade conduz a organização como um todo, e em especial a Unidade de Informação, a um processo de melhoria contínua e integrada, em direção ao cumprimento de sua missão.

A gestão pela qualidade é desenvolvida atualmente através de três abordagens mundiais 
distintas sendo:

\begin{tabular}{|c|c|}
\hline ABORDAGEM & PRINCÍPIO \\
\hline Americana & $\begin{array}{c}\text { Qualidade não acontece } \\
\text { por acaso; Baseada na } \\
\text { Trilogia de Juran® } \\
\text { (planejamento, controle e } \\
\text { aperfeiçoamento) }\end{array}$ \\
\hline Japonesa & $\begin{array}{c}\text { Fundamenta-se em } \\
\text { métodos estatísticos e na } \\
\text { valorização do ser } \\
\text { humano; Baseada nos } \\
\text { princípios de Deming }\end{array}$ \\
\hline Européia & $\begin{array}{c}\text { Qualidade é } \\
\text { padronização dos } \\
\text { processos; Baseada nas } \\
\text { normas ISO 9000 }\end{array}$ \\
\hline
\end{tabular}

A abordagem européia foi introduzida no Brasil através da criação do Sistema Brasileiro de Qualidade e Produtividade (PBQP), em 1990, com o objetivo de produzir mudanças estruturais que contemplaram, entre outros fatores, a criação do Sistema Brasileiro de Certificação (SBC), com o fim de aumentar o número de produtos e serviços certificados e promover o reconhecimento internacional destes.

As Normas ISO 9000 apresentam três opções básicas de modelos e de garantia pela qualidade, variando entre o menos (ISO 9003) e o mais (ISO 9001) abrangente, ficando clara a necessidade de "inclusão de dispositivos adequados para a devida identificação, coleta, indexação, arquivamento e disposição de informações e documentação para o sistema da qualidade" (ISO 9004, 1990).

\section{Processos de Certificação}

Uma vez que a organização adota e implanta qualquer um dos trềs níveis, selecionado de acordo com o que ela deseja passar ao seu cliente em relação à qualidade daquilo que fornece, o procedimento seguinte é atestar a conformidade dos produtos ou serviços aos padrões e especificações pré-estabelecidos, ou seja, é validar qualitativamente o processo de desenvolvimento, produção e distribuição dos produtos e serviços colocados à disposição do cliente. Este procedimento chama-se certificação.

A adoção das normas da Família ISO 9000, pelos organismos normativos nacionais, ocasionou um desenvolvimento na Certificação, que consiste na Avaliação de Conformidade do Sistema de Qualidade da organização. (SEBRAE/ ABNT 1993).

Isto implica na adequação da organização como um todo às exigências das normas, e não na adequação dos bens ou serviços em si. Não se trata de avaliar os bens ou serviços, nem de se realizar controles sobre os mesmos.

A Certificação tem por objetivo demonstrar a capacidade da organização para produzir bens ou serviços em conformidade com as exigências, ou seja, é o reconhecimento de que a organização possui um sistema de qualidade. Ela funciona como um fator de marketing uma vez que assegura bens e serviços de qualidade aos clientes.

O Certificado de Conformidade é um documento emitido de acordo com as regras de um sistema de certificação, indicando existir um nível adequado de confiança de que bens, processos ou serviços devidamente identificados estão em conformidade com uma norma específica.

De acordo com o SEBRAE/ABNT (1993), as vantagens da certificação são:

a) registro da organização;

b) benefícios para a implantação de sistemas de garantia pela qualidade e, portanto, prestação de serviços e fabricação de bens mais competitivos;

c) possibilita a assinatura de acordos de reconhecimento de marcas e certificados;

d) facilita e barateia a certificação de bens;

e) facilita a implantação pela qualidade total nas organizações;

f) evita avaliações de diferentes clientes e fornecedores; $\mathrm{e}$

g) aumenta os argumentos comerciais.

\section{CERTIFICAÇÃO DAS UNIDADES DE INFORMAÇÃ O}

A certificação é um processo de avaliação catalisador de mudanças, e pode ser útil para unidades de informação, adotado em outras áreas pela necessidade de se manter a qualidade através do uso de normas pré-estabelecidas.

O processo de certificação de Unidades de Informação tem como objetivo adequar as 
necessidades dos usuários ao provimento de serviços prestados, contribuir para a melhoria dos serviços de informação, descrever métodos estruturados de medição da qualidade através de um sistema de pontuação.

Estes processos de medições poderão, segundo Whitehall (1992), estabelecer processos de melhoria a partir de critérios específicos através de medidas de acessibilidade, de cobertura e relevância da coleção e tempo de resposta.

Uma lista elaborada por Potter (1985) inclui como critérios de qualidade a localização, horário de funcionamento e layout da Unidade de Informação.

O primeiro esforço de certificação de serviços prestados por Unidades de Informação tem origem nos pré-requisitos estabelecidos pelo Canadian Council on Health publicado em 1990 contemplando a análise de: objetivos e metas da unidade de informação, organização e administração, direção e recursos humanos, facilidades, equipamentos e suprimentos, políticas e procedimentos, serviços e recursos da informação, educação e sistema de garantia da qualidade (Brockman, 1992)

Recentemente, a Inglaterra adotou um sistema de certificação de Unidades de Informação (Hewlett, 1994) na área de ensino da saúde com o objetivo de garantir a informação precisa e aperfeiçoada e servir de catalisador de mudanças para as Unidades de Informação inglesas.

\section{Sistema de Certificação Inglês}

Este sistema surgiu da necessidade da distribuição mais adequada de verbas, de estabelecer prioridades para o sistema educacional, a fim de possibilitar a cooperação entre as bibliotecas das região onde o sistema de certificação foi implantado, além de coordenar estatisticamente informações que são compiladas para uma bibliografia nacional. níveis:

As certificações são realizadas em três

1o. Nível - quando todos os elementos das normas de certificação são aplicados;

2o. Nível - quando os pontos essenciais estabelecidos pelas normas são aplicados e onde há evidências de um processo contínuo de melhoria em direção ao nível 1;

3o. Nível - quando a aplicação dos pontos essenciais exigem a cooperação de bibliotecas maiores para subsidiar os serviços e que haja um progresso contínuo de melhorias para o nível 2.

À princípio, todas as Unidades de Informação integrantes do sistema são certificadas no nível 3 e, com o preenchimento do instrumento de avaliação e posterior visita do Comitê Certificador, passam a possuir níveis diferenciados conforme as análises realizadas, servindo de estímulo para a melhoria contínua dos serviços desenvolvidos.

Os pontos avaliados para certificação fazem parte de uma Lista de Padrões Mínimos que o Comitê considera existirem nas Unidades de Informação que fazem parte do sistema (os questionamentos são apresentados sempre de forma afirmativa). Esta lista é composta de:

\section{FILOSOFIA E ADMINISTRAÇÃO}

1.1 Metas e objetivos escritos que foram preparados em colaboração com representantes da comunidade e de usuários;

1.2 Revisões anuais em metas e objetivos com vista a acompanhar as mudanças e para identificar a necessidade do crescimento da Biblioteca (inclusive de pessoal) aqueles responsáveis pelo orçamento/recursos e para a administração como um todo;

1.3 A Biblioteca oferece serviço a todos os grupos de pessoas dentro de sua instituição e distritos que fazem uma contribuição formal para a manutenção da Biblioteca;

1.4 A Biblioteca fica aberta e tem um corpo de funcionários qualificados para funcionar por um período suficiente para atender a maioria de seus leitores;

1.5 Existe um processo formalizado de consulta regular à comunidade de usuários (isso pode ser feito através de um "Comitê de Bibliotecas";

1.6 O bibliotecário desempenha uma participação ativa nesse processo formal de consulta;

1.7 Registro do processo de consulta é mantido; 
1.8 Onde o serviço da Biblioteca emprega diversos funcionários, são mantidas reuniões regulares;

1.9 A Biblioteca mantém estatísticas que irá capacita-la a contribuir para os registros estatísticos para o Grupo Regional;

1.10 As estatísticas da Biblioteca são usadas no processo indicado no item 1.2;

1.11 A Biblioteca mentem um manual de políticas e procedimentos relacionados às suas atividades. Este manual está disponível para consulta a qualquer hora pelos funcionários da Biblioteca;

$1.12 \mathrm{O}$ manual da Biblioteca é revisado regularmente assim que os procedimentos mudam;

1.13 A Biblioteca tem um programa atualizado de garantia da qualidade disponível para elementos essenciais de seus serviços;

1.14 A Biblioteca estimula seus usuários a fazerem sugestões sobre seus serviços e facilidades físicas (isso pode tomar a forma de Caixa de Sugestão);

1.15 Existe uma estrutura organizacional atual e escrita que identifica as linhas de administração e responsabilidades;

1.16 Os funcionários da Biblioteca estão conscientes da legislação, políticas de seus empregadores e das suas obrigações com relação a:

- Direitos Autorais;

- Proteção de Dados;

- Saúde e Segurança;

\section{ACOMODAÇÃO E EQUIPAMENTOS}

2.1 A acomodação da Biblioteca serve ao seu usuário com coleções que estão em livre acesso e prontamente acessível a todos os seus leitores. Isso leva em conta as necessidades de funcionários e usuários deficientes;

2.2 A Biblioteca tem uma área adequadamente arranjada para estudos silenciosos;
2.3 A área da Biblioteca foi arranjada de tal modo a otimizar a segurança de suas coleções e recursos;

2.4 A saúde e segurança de ambos (funcionários e usuários) foi considerada no desenho da área física da Biblioteca. Isso leva em conta algumas questões como: iluminação, ventilação e segurança geral e isso se enquadra aos padrões apropriados;

2.5 A área da Biblioteca é usada somente com objetivos biblioteconômicos;

2.6 Há números adequados de espaços de estudos para os leitores da Biblioteca com acomodações variadas por exemplo cabinas, mesas de estudo, poltronas, mezinhas, assentos adequados para micros e equipamentos audiovisual além de acomodações adequadas para obras de referências;

2.7 A Biblioteca tem espaço/prateleiras adequado para o seu estoque e tem um nível razoável de espaço para expansão. Isso incluirá uma área para exposição de periódicos correntes e prateleiras adequadas para livros, periódicos, obras de referência, índices ou material audiovisual;

2.8 A Biblioteca oferece um ponto facilmente identificável pelo usuário (isso pode tomar forma de um Balcão de Informações);

2.9 O bibliotecário tem escritório/sala de trabalho e espaço de trabalho para lidar com a aquisição, catalogação, processamento de livro, preparação para encadernação ou outras tarefas relacionadas;

2.10 Uma Sala de Seminários está disponível para uso da Biblioteca;

2.11 A Biblioteca tem equipamento/mobília geral de escritório apropriada ao seu tamanho e função;

2.12 Os usuários da Biblioteca tem acesso fácil a uma máquina de xerox;

\subsection{A Biblioteca tem um telefone;}

2.14 Instalações apropriadas estão disponíveis para usos de itens audiovisuais incluindo: VídeoCassete, AudioTape e Slides;

$2.15 \mathrm{O}$ equipamento da Biblioteca é mantido adequadamente;

2.16 A Biblioteca tem uma sinalização interna 
efetiva;

2.17 O caminho até a Biblioteca é adequadamente sinalizado;

2.18 A área disponível para a Biblioteca está de acordo com o padrão apropriado, por exemplo Nota 42 sobre Construção de Edifícios Hospitalares, Acomodação Para Educação e Treinamento2 .

\section{COLEÇÃO DA BIBLIOTECA}

3.1 A Biblioteca tem o estoque adequado de livros e relatórios relevantes para cada disciplina/ área de especialização representada no Hospital/ Distrito (um padrão sugerido é a MIWP Hague/ Jackson Core List)3 Isto inclui o material que apoia o desenvolvimento e necessidades gerenciais de seus usuários;

3.2 Os procedimentos são executados para assegurar que as coleções da Biblioteca permaneçam bem equilibradas, relevantes e atualizadas;

3.3 O tamanho do estoque de livros é apropriado para o número de usuários da Biblioteca;

3.4 Os usuários são encorajados a colaborar com os bibliotecários na seleção das obras;

3.5 Há um catálogo da coleção completa por autor, título e assunto;

3.6 Um esquema reconhecido de classificação é utilizado;

3.7 O estoque de livros é arrumado nas estantes por ordem de classificação;

3.8 A coleção de livros é tão sinalizada e organizada que os usuários são capazes de encontrar o material sem assistência dos funcionários da Biblioteca;

3.9 Uma proporção significativa do estoque de livros é disponível para empréstimo;

3.10 A Biblioteca tem uma coleção apropriada de livros de referências gerais e especializadas (dicionários, guias, anuários etc);

3.11 A Biblioteca assina periódicos correntes relevantes a cada disciplina ou área representada no trabalho do Hospital/Distrito (Normas da MIWP Hague/Jackson Core List);

3.12 Há uma lista de todos os periódicos da Biblioteca disponível para o usuário;

3.13 A Biblioteca assina o Index Medicus em qualquer uma de suas formas, por exemplo o condensado, CD-ROM etc (Isto não inclui o acesso on-line ou Medline);

3.14 A Biblioteca assina outros serviços relevantes de indexação ou "abstracting";

3.15 A Biblioteca tem coleções apropriadas de meios não gráficos, por exemplo slides, vídeocassete, audiotape.

\section{FINANÇAS}

4.1 O Bibliotecário participa no estabelecimento do financiamento da Biblioteca. Isto é decidido anualmente e leva em conta:

I - Aumentos antecipados nos custos de livros e periódicos e outros itens da Biblioteca

II - Os materiais sugeridos assim identificados em listas tais como MIWP Hague/Jackson Core List;

4.2 Os itens do orçamento da Biblioteca devem incluir pelo menos: staff, treinamento de staff, periódico, livros, materiais audiovisuais, serviços de computação, serviços dos usuários;

4.3 O Bibliotecário supervisiona o gasto do orçamento da Biblioteca;

4.4 A Biblioteca recebe relatórios regulares precisos, detalhados e atualizados sobre os gastos ao longo do ano fiscal;

\section{RECURSOS HUMANOS}

5.1 O serviço bibliotecário é supervisionado por um bibliotecário adequadamente qualificado e com experiência;

5.2 A devida consideração é dada pelos selecionadores ao papel da qualificação profissional em Biblioteconomia; 
5.3 Para as Bibliotecas menores dentro de um serviço bibliotecário diversificado, um bibliotecário com adequada experiência e qualificação está acessível aos usuários da Biblioteca;

5.4 A devida consideração é dada pelos gerentes às diretrizes salariais estabelecidas pela Associação de Bibliotecários;

5.5 Há um sistema de avaliação em andamento para os funcionários da Biblioteca;

5.6 Oportunidades de afastamento para estudos para a educação continuada estão disponíveis para os usuários da Biblioteca;

5.7 O corpo de funcionários se engaja nas atividades e segue as diretrizes da Associação de Bibliotecários sobre Desenvolvimento Profissional Continuado;

5.8 Os funcionários da Biblioteca são encorajados a contribuir para as atividades das Associações Profissionais das quais eles são membros;

5.9 Há descrição de funções e contrato de emprego escritos para cada causa na Biblioteca;

5.10 Os novos contratados para o serviço bibliotecário são envolvidos num processo formal de indução que os introduzem aos aspectos relevantes da organização / instituição no qual eles irão trabalhar.

\section{SERVIÇOS BÁSICOS}

6.1 A Biblioteca oferece serviço de empréstimo, renovação e reserva;

6.2 A Biblioteca oferece um serviço de Pergunta e Resposta. Isto incorpora outro serviço referencial a outros provedores de informação quando for apropriado;

6.3 A Biblioteca tem um programa de educação do usuário:

a) para introduzir novos leitores aos serviços da Biblioteca

b) para passar habilidade de longo prazo no manuseio da informação

c) para introduzir novos serviços e facilitadores para seus leitores;

$6.4 \mathrm{O}$ serviço bibliotecário é adequadamente divulgado e há um guia impresso disponível;

6.5 A Biblioteca oferece um serviço de atendimento entre Bibliotecas e as fotocópias oferecidas como parte desse serviço podem ser retiradas por seus leitores dentro das diretrizes, Lei de Direitos Autorais;

6.6 A Biblioteca oferece um serviço de alerta adequado e corrente na forma de lista de Livros Especializados ou Arranjos de Alerta da Literatura.

\section{COOPERAÇÃO ENTRE BIBLIOTECAS}

7.1 A Biblioteca participa de empréstimo entre Bibliotecas de área da saúde regional e outras redes cooperativas;

7.2 A Biblioteca usa o serviço de fornecimento de documento da British Library;

7.3 A Biblioteca tem arranjos apropriados com outras Bibliotecas e Centros de Informação, que iriam estender os recursos disponíveis aos seus usuários (Ex: BMA, Royal Colleges etc);

7.4 O bibliotecário tem conhecimento das facilidades e serviços que estão disponíveis via o N. E. Themes Regional Library Service e o Bibliotecário Regional.

\section{TECNOLOGIAS DA INFORMAÇÃO}

8.1 A Biblioteca tem um sistema de manutenção interno incluindo circulação e catalogação;

8.2 Um computador está disponível para o uso dos funcionários da Biblioteca;

8.3 A Biblioteca oferece facilidades para a busca on-line de bancos de dados computadorizados (isto pode ser via outra Biblioteca);

8.4 A Biblioteca tem um sistema de CD-ROM de acesso ao MedLine e outras bases de dados relacionadas a área de saúde;

8.5 Um fax está disponível para entrega de documentos;

8.6 A Biblioteca tem acesso as fontes de informação automatizadas internas do Hospital. 
digna.

\section{CONCLUSÕES}

As Unidades de Informação, como organismos fornecedores de informação, possuem um caráter próprio: prestadoras de serviço. Isto implica em uma relação complexa com seus clientes uma vez que serviços de informação são intangíveis (não podem ser medidos tão facilmente), heterogêneos (seu desempenho varia de acordo com o comportamento do cliente) e simultâneos (a execução e o consumo são praticamente realizados ao mesmo tempo).

Isto implica, segundo Parasuraman, Zeithaml \& Berry (1985), na necessidade da criação de um modelo de gestão pela qualidade que apresente dimensões distintas exigidas pelo ambiente onde atuam. Estas dimensões estão relacionadas a qualidade técnica (capacidade de manter serviços atualizados), qualidade funcional (capacidade de envolvimento total da instituição), qualidade física (relacionada a equipamentos e estrutura física), qualidade corporativa (relacionada a imagem que a instituição possui no mercado) e qualidade interativa (capacidade de envolvimento entre os fornecedores direto dos serviços e os consumidores).

Com a adoção da qualidade por organizações de serviços, e especialmente pelas Unidades de Informação, a melhoria do desempenho do serviço, da satisfação do cliente, da produtividade e da eficiência torna-se fator essencial para o aumento de seu mercado de atuação.

Isto implica na efetiva utilização de procedimentos e instrumentos que permitam a padronização de seu desempenho de forma a produzir serviços qualitativos e garantir a total satisfação das expectativas e necessidades dos clientes. Isto é possível através da aplicação de certificação de conformidade, apresentado nesse trabalho com objetivo de possibilitar uma avaliação conjuntural para o cenário brasileiro em função do que se apresenta no cenário mundial.

A busca pela excelência de seus serviços torna as Unidades de Informação cumpridoras do papel que representam perante a sociedade. Ser disseminadora de informações que permitam a construção de uma cidadania mais justa e mais

\section{REFERÊNCIAS BIBLIOGRÁFICAS}

BROCKMAN, John R. Just another management fad?: the implication of TQM for library and information services. ASLIB Proceeding, Londres, v.44, n.7/8, p.283-288, Jul./Aug. 1992.

HEWLETT, John. Quality control in library information services. In: SEMINÁRIO INTERNACIONAL SOBRE OS DESAFIOS DA ERA DA INFORMAÇÃO, 1994, São Paulo. Anais... São Paulo: BIREME, 1994. p.1-8.

PARASURAMAN, A., ZEITHAML, Valarie A., BERRY, Leonard L. A conceptual model of service quality and its implications for future research. Journal of Marketing, v.49, n.4, p.41-50, Fall, 1985.

POTTER, J. Performance measures: the user view. In: HARRIS, C., CLIFFORD, B. Public libraries: re-appraisal and restructuring. London, 1985. p. 111-29.

SERVIÇO DE APOIO À MICRO E PEQUENAS EMPRESAS DE SÃO PAULO/ ASSOCIAÇÃO BRASILEIRA DE NORMAS TÉCNICAS. Seminário regional: NBR / ISO 9000 visão tecnológica de incremento à competitividade. 1993, Campinas: SEBRAE/ABNT, 1993. (Anotações)

WHITEHALL, Tom. Quality in library and information services: a review. Library Management, v.13, n.5, p.23-35, 1992. 
Célia Regina Simonetti Barbalho

Professora de Administração de Bibliotecas na Universidade Federal do Amazonas - UFAM. Mestre em Biblioteconomia pela PUCCAMP. Doutoranda em Comunicação e Semiótica - PUC/ SP.

\section{Title}

Pattern of conformity for Information Units: the presentattion of the English model

\section{Abstract}

This work presents the parameters for quality accreditation established by the National Health Service in Great Britain.

\section{Keyworlds}

Quality - Accreditation

Apresentado no COBIBiii em agosto de 1995 